\title{
Systemic Image-Guided Liver Cancer Radiovirotherapy Using Dendrimer-Coated Adenovirus Encoding the Sodium Iodide Symporter as Theranostic Gene
}

\author{
Geoffrey K. Grünwald ${ }^{1}$, Alexandra Vetter ${ }^{2}$, Kathrin Klutz ${ }^{1}$, Michael J. Willhauck ${ }^{1}$, Nathalie Schwenk ${ }^{1}$, \\ Reingard Senekowitsch-Schmidtke ${ }^{3}$, Markus Schwaiger ${ }^{3}$, Christian Zach $^{4}$, Ernst Wagner ${ }^{2}$, Burkhard Göke ${ }^{1}$, \\ Per S. Holm ${ }^{5}$, Manfred Ogris ${ }^{2}$, and Christine Spitzweg ${ }^{1}$ \\ ${ }^{1}$ Department of Internal Medicine II-Campus Grosshadern, University Hospital of Munich, Munich, Germany; ${ }^{2}$ Pharmaceutical \\ Biotechnology, Department of Pharmacy, Center for System-Based Drug Research, and Center for Nanoscience, Ludwig-Maximilians \\ University, Munich, Germany; ${ }^{3}$ Department of Nuclear Medicine, University Hospital Klinikum rechts der Isar, Munich, Germany; \\ ${ }^{4}$ Department of Nuclear Medicine, University Hospital of Munich, Munich, Germany; and ${ }^{5}$ Institute of Experimental Oncology, \\ University Hospital Klinikum rechts der Isar, Munich, Germany
}

Currently, major limitations for the clinical application of adenovirus-mediated gene therapy are high prevalence of neutralizing antibodies, widespread expression of the coxsackie-adenovirus receptor (CAR), and adenovirus sequestration by the liver. In the current study, we used the sodium iodide symporter (NIS) as a theranostic gene to investigate whether coating of adenovirus with synthetic dendrimers could be useful to overcome these hurdles in order to develop adenoviral vectors for combination of systemic oncolytic virotherapy and NIS-mediated radiotherapy. Methods: We coated replication-deficient (Ad5-CMV/NIS) (CMV is cytomegalovirus) and replication-selective (Ad5-E1/AFP-E3/NIS) adenovirus serotype 5 carrying the $h$ NIS gene with poly(amidoamine) dendrimers generation 5 (PAMAM-G5) in order to investigate transduction efficacy and altered tropism of these coated virus particles by ${ }^{123} \mid$ scintigraphy and to evaluate their therapeutic potential for systemic radiovirotherapy in a liver cancer xenograft mouse model. Results: After dendrimer coating, Ad5-CMV/NIS demonstrated partial protection from neutralizing antibodies and enhanced transduction efficacy in CAR-negative cells in vitro. In vivo ${ }^{123}$ | scintigraphy of nude mice revealed significantly reduced levels of hepatic transgene expression after intravenous injection of dendrimer-coated Ad5-CMV/NIS (dcAd5-CMV/NIS). Evasion from liver accumulation resulted in significantly reduced liver toxicity and increased transduction efficiency of dcAd5-CMV/NIS in hepatoma xenografts. After PAMAM-G5 coating of the replication-selective Ad5-E1/AFP-E3/NIS, a significantly enhanced oncolytic effect was observed after intravenous application (virotherapy) that was further increased by additional treatment with a therapeutic dose of ${ }^{131}$ I (radiovirotherapy) and was associated with markedly improved survival. Conclusion: These results demonstrate efficient liver detargeting and tumor retargeting of adenoviral vectors after coating with synthetic dendrimers, thereby representing

Received Oct. 10, 2012; revision accepted Feb. 26, 2013.

For correspondence or reprints contact either of the following: Christine Spitzweg, Medizinische Klinik II-Campus Grosshadern, Klinikum der Universität München, Marchioninistrasse 15, 81377 Munich, Germany.

E-mail: Christine.Spitzweg@med.uni-muenchen.de

Manfred Ogris, Department of Pharmacy, Center of Drug Research, Pharmaceutical Biotechnology, Butenandtstrasse 5-13, 81377 Munich, Germany.

E-mail: Manfred.Ogris@cup.uni-muenchen.de

Published online Jul. 10, 2013.

COPYRIGHT (C 2013 by the Society of Nuclear Medicine and Molecular Imaging, Inc. a promising innovative strategy for systemic NIS gene therapy. Moreover, our study - based on the function of NIS as a theranostic gene allowing the noninvasive imaging of NIS expression by 123| scintigraphy-provides detailed characterization of in vivo vector biodistribution and localization, level, and duration of transgene expression, essential prerequisites for exact planning and monitoring of clinical gene therapy trials that aim to individualize the NIS gene therapy concept.

Key Words: adenovirus serotype 5; PAMAM dendrimer; sodium iodide symporter (NIS); radiovirotherapy; gene therapy

J Nucl Med 2013; 54:1450-1457

DOI: 10.2967/jnumed.112.115493

$\mathbf{G}$ enetically engineered replication-selective adenoviruses represent efficient gene transfer vehicles with the advantage of potentiating therapeutic efficacy of gene therapy by its own oncolytic activity (1). Because of limited virus spread in the tumor, virotherapy is ideally combined with therapeutic genes that are associated with a bystander effect. In its well-characterized dual function as reporter and therapy gene associated with a significant physical bystander effect, the sodium iodide symporter (NIS) represents an ideal candidate gene for replication-selective adenovirusmediated gene virotherapy. It provides the possibility of detailed noninvasive monitoring of biodistribution of virus infection and replication in addition to stimulation of therapeutic efficacy of oncolytic virotherapy by additional NIS-mediated radionuclide therapy $(2,3)$.

However, recombinant adenoviruses still face hurdles that strongly limit efficient and safe application, in particular for systemic gene delivery, including induction of immune and inflammatory responses, elimination by neutralizing antibodies, high promiscuity due to widespread expression of the coxsackieadenovirus receptor (CAR), and significant pooling in the liver (4). Besides genetic engineering (5), chemical modification of the adenovirus' surface represents a convenient method to shield the virus from undesired interactions with blood components and allows its retargeting to tumor cells lacking adenoviral receptors (4). Therefore, different strategies have been developed in order to combine 
viral and synthetic vectors into a hybrid vector, including covalent conjugation of reactive polymers based on polyethylene glycol or $\mathrm{N}$-[2-hydroxypropyl]methacrylamide (6) and noncovalent modification of the negatively charged adenovirus surface by electrostatic interaction with cationic polymers (5). These new technologies demonstrated first evidence to provide the vector with the ability to overcome neutralizing antivector antibodies, to escape liver tropism and to reduce innate and adaptive immune responses and liver toxicity even after systemic vector application, while maintaining its natural biologic activity (6).

We have recently used chemically well-defined dendritic PAMAM (poly(amidoamine)) dendrimers bearing positively charged terminal amines to coat the negatively charged adenoviral capsid by virtue of electrostatic interaction (7). This modification allowed efficient internalization and transduction of tumor cells in vitro otherwise refractory toward adenoviral transduction.

In the present study, we used this technology for coating of adenovirus vectors carrying the $h$ NIS gene, followed by the analysis of altered transduction efficiency, biodistribution, vectorrelated toxicity, and therapeutic potential after systemic adenovirusmediated NIS gene delivery. On the basis of its function as a theranostic gene, NIS was used for the noninvasive imaging of biodistribution and transduction efficiency by ${ }^{123}$ I scintigraphy. The evaluation of tumor-specific oncolytic efficacy (virotherapy) was followed by the assessment of NIS-mediated therapy response after the application of an additional therapeutic dose of ${ }^{131} \mathrm{I}$ (radiovirotherapy).

\section{MATERIALS AND METHODS}

\section{Cell Culture}

The human cell lines HuH7 (HCC, JCRB 0403 [Japanese Collection of Research Bioresources Cell Bank, National Institute of Health Sciences]), SKOV-3 (ovarian carcinoma, HTB-77 [American Type Culture Collection]), and U87 MG (glioblastoma, HTB-14 [American Type Culture Collection]) were cultured as described previously (7). Cellular CAR levels were analyzed by flow cytometry as described previously (7).

\section{Production and Dendrimer Coating of Recombinant Adenovirus}

The replication-deficient adenovirus Ad5-CMV/NIS $\left(1.1 \times 10^{12}\right.$ particles $\left.=5.0 \times 10^{10} \mathrm{PFU}\right)$ carrying the $h$ NIS complementary DNA under the control of the unspecific cytomegalovirus (CMV) promoter $(8)$ and the replication-selective adenovirus Ad5-E1/AFP-E3/ NIS $\left(1.1 \times 10^{12}\right.$ particles $\left.=3.0 \times 10^{10} \mathrm{PFU}\right)$ carrying the human NIS gene linked to the mouse $\alpha$-fetoprotein promoter (3) were developed as described previously. Amine-terminated generation 5 PAMAM dendrimer was used as described previously (7). For surface modification, the adenovirus was dispensed with serum-free OPTI-MEM (Invitrogen) to a volume of $50 \mu \mathrm{L}$ (in vitro experiments) or $125 \mu \mathrm{L}$ (in vivo experiments) and gently mixed with an equal volume of PAMAM-G5 (10 or $300 \mathrm{ng}$ ) diluted in HEPES ( $N$-(2-hydroxyethyl) piperazine- $N^{\prime}$-(2-ethanesulfonic acid))-buffered glucose $(20 \mathrm{mmol} / 1$ HEPES, $5 \%$ glucose $[\mathrm{w} / \mathrm{v}]$, ph 7.4). The mixture was incubated at room temperature for $30 \mathrm{~min}$ before use. Dendrimer coating of the virus with $10 \mathrm{ng}$ of PAMAM-G5 is indicated by the prefix $\mathrm{dc}_{10}$ and with $300 \mathrm{ng}$ of PAMAM-G5 by the prefix $\mathrm{dc}_{300}$.

\section{In Vitro NIS Gene Delivery}

Twenty-four hours after seeding $\left(1.5 \times 10^{5}\right.$ cells/well in 12 -well plates), cells were incubated with increasing MOI (multiplicity of infection) of Ad5-CMV/NIS, $\mathrm{dc}_{10} \mathrm{Ad5-CMV/NIS,} \mathrm{or} \mathrm{dc}_{300} \mathrm{Ad5}-$
CMV/NIS per well in serum-free OPTI-MEM for $45 \mathrm{~min}$ at $37^{\circ} \mathrm{C}$. The medium was replaced by fresh culture medium, and virusinfected cells were further maintained for $4 \mathrm{~d}$ before iodide uptake was measured at steady-state conditions as described previously (9). Results were normalized to cell viability as described previously (10) and expressed as cpm/A $490 \mathrm{~nm}$.

For in vitro neutralization experiments, Ad5-CMV/NIS or dc 300 Ad5CMV/NIS was incubated for $30 \mathrm{~min}$ with increasing amounts of polyvalent adenovirus-neutralizing IgG (Privigen; CSL Behring) at room temperature. The final concentration of $\mathrm{IgG}$ was between 0.1 and $3.0 \mathrm{mg} / \mathrm{mL}$ as indicated. HuH7 cells were infected with virus at 100 MOI per well and analyzed for iodide uptake activity.

\section{In Vivo NIS Gene Transfer and Biodistribution Imaging Studies}

For proof of principle of systemic NIS gene transfer using a dendrimer-coated adenovirus, the $\mathrm{HuH7}$ xenograft mouse model was chosen that had already been used in our previous studies and that was established as outlined previously (3). The experimental protocol was approved by the regional governmental commission for animals (Regierung von Oberbayern, Munich, Germany). Experiments started when tumors had reached 7-9 mm ( ${ }^{123}$ I biodistribution studies) or 4-5 $\mathrm{mm}\left({ }^{131} \mathrm{I}\right.$ radiovirotherapy studies). After a 10 -d pretreatment with $\mathrm{L}-$ T4 (L-thyroxine Henning; Sanofi-Aventis) $(5 \mathrm{mg} / \mathrm{L})$ in their drinking water to reduce iodide uptake by the thyroid gland and maximize radioiodine uptake in the tumor, animals were injected intravenously via the tail vein with $1 \times 10^{9} \mathrm{PFU}$ of the respective adenovirus.

Four days after systemic adenovirus injection, mice received 18.5 $\mathrm{MBq}$ of ${ }^{123} \mathrm{I}$ intraperitoneally, and radioiodine biodistribution was monitored by serial $\gamma$-camera imaging as described previously (11). Regions of interest in the liver were quantified and expressed as a fraction of the amount of accumulated radionuclide in the livers of mice injected with the unmodified Ad5-CMV/NIS. Regions of interest in the tumor were quantified and expressed as a fraction of the total amount of applied radioiodine per gram of tumor tissue (percentage injected dose per gram $[\% \mathrm{ID} / \mathrm{g}])$. The retention time within the tumor was determined by serial scanning after radioiodine injection, and dosimetric calculations were performed according to the concept of MIRD, with the dose factor as determined by RADAR (RAdiation Dose Assessment Resource) (www.doseinfo-radar.com).

For serial iodide uptake studies, mice were imaged for ${ }^{123} \mathrm{I}$ biodistribution on the indicated days after intravenous administration of Ad5-E1/AFP-E3/NIS or dc $_{300}$ Ad5-E1/AFP-E3/NIS. Regions of interest in the tumor were quantified and expressed as \%ID per $100 \mathrm{~mm}^{3}$ of tumor tissue $\left(\% \mathrm{ID} / 100 \mathrm{~mm}^{3}\right)$.

\section{Ex Vivo Analysis}

After systemic adenovirus injection, NIS messenger RNA (mRNA) expression levels of liver, lung, spleen, kidneys, and tumors were analyzed via quantitative real-time PCR (qPCR) as described previously (12). Immunofluorescence staining of paraffin-embedded tissue sections derived from livers and $\mathrm{HuH} 7$ xenografts was performed using an $h$ NIS-specific antibody directed against amino acid residues 625-643 of human NIS (Millipore) at a dilution of 1:750. Four days after systemic adenovirus injection, mice were sacrificed, and blood serum samples were collected to assess alanine aminotransferase and aspartate aminotransferase levels (measured at the Department of Clinical Biochemistry and Pathobiochemistry, Klinikum rechts der Isar, Munich, Germany). Subsequently, liver tissues were harvested and embedded in paraffin for hematoxylin and eosin staining.

\section{Radiovirotherapy Study}

$\mathrm{HuH7}$ xenografts were established in 5 groups of mice. The first group was injected intravenously with saline only $(\mathrm{NaCl}$ control). A 
second group received a single intravenous injection of $1 \times 10^{9} \mathrm{PFU}$ of the conditionally replicating Ad5-E1/AFP-E3/NIS, and a third group received $1 \times 10^{9} \mathrm{PFU}$ of $\mathrm{dc}_{300} \mathrm{Ad} 5$-E1/AFP-E3/NIS. The fourth group received $1 \times 10^{9} \mathrm{PFU}$ of Ad5-E1/AFP-E3/NIS and $3 \mathrm{~d}$ later a single intraperitoneal dose of $55.5 \mathrm{MBq}$ of ${ }^{131} \mathrm{I}$. And the fifth group received $1 \times 10^{9} \mathrm{PFU}$ of $\mathrm{dc}_{300} \mathrm{Ad} 5$-E1/AFP-E3/NIS and $3 \mathrm{~d}$ later 55.5 $\mathrm{MBq}$ of ${ }^{131} \mathrm{I}$. Tumors were measured twice weekly thereafter. Mice were followed for $70 \mathrm{~d}$ or until tumor burden was such that animals had to be killed $\left(\geq 1,500 \mathrm{~mm}^{3}\right)$.

\section{Statistical Methods}

All in vitro experiments were performed in triplicate. Results are represented as mean $\pm \mathrm{SD}$ of triplicates. Statistical significance was tested using a Student $t$ test $(* P \leq 0.05 ; * * P \leq 0.01 ; * * * P \leq 0.001)$. Statistical significance of in vivo experiments was calculated using the Mann-Whitney $U$ test (2-tailed).

\section{RESULTS}

\section{Influence of Dendrimer Coating In Vitro}

Transfection efficacy of uncoated Ad5-CMV/NIS correlated well with levels of CAR expression (Figs. 1A-1C; Supplemental Fig. 1 [supplemental materials are available online only at http:// jnm.snmjournals.org]). CAR-positive HuH7 cells showed an MOIdependent increase in perchlorate-sensitive ${ }^{125} \mathrm{I}$ accumulation of up to 70 -fold as compared with uninfected cells, which was significantly increased after infection with $\mathrm{dc}_{10} \mathrm{Ad} 5-\mathrm{CMV} / \mathrm{NIS}$ and further retained after infection with $\mathrm{dc}_{300} \mathrm{Ad5}$-CMV/NIS (Fig. 1A). The low-CAR-level cell line U87 MG showed low transduction efficacy when incubated with Ad5-CMV/NIS. In contrast, U87 MG cells treated with $\mathrm{dc}_{10} \mathrm{Ad5-CMV/NIS}$ or $\mathrm{dc}_{300} \mathrm{Ad5}$ CMV/NIS (Fig. 1B) showed an up to 5.5-fold increase in iodide uptake activity. The CAR-negative SKOV-3 cells showed no iodide accumulation above background level, even when incubated with a high MOI of Ad5-CMV/NIS. Coating of Ad5-CMV/NIS led to an up to 22-fold increase in iodide uptake activity (Fig. 1C). NIS gene transfer did not alter cell viability (data not shown).

Ad5-CMV/NIS was rapidly neutralized by increasing amounts of human IgG solution. In contrast, $\mathrm{dc}_{300} \mathrm{Ad5}$-CMV/NIS showed partial protection from neutralizing antibodies as demonstrated by a decelerated decrease in iodide uptake activity (Fig. 1D).

\section{Virus Biodistribution and Toxicity Studies In Vivo}

In vivo experiments showed high levels of radioiodine accumulation in the liver of tumor-free mice after systemic injection of Ad5-CMV/NIS $(n=5)$ due to hepatic pooling of the vector as shown by ${ }^{123} \mathrm{I} \gamma$-camera imaging $6 \mathrm{~h}$ after injection (Fig. 2A, left), which was significantly (up to $70 \%$ ) lower after systemic injection of virus particles coated with increasing amounts (10 ng, $n=5$; $300 \mathrm{ng}, n=5$ ) of PAMAM-G5 (Fig. 2A, right; Supplemental Fig. 2A). Significant radioiodine accumulation was also observed in tissues physiologically expressing NIS, including the stomach and thyroid, as well as in the urinary bladder due to renal elimination of the radionuclide. The results were confirmed by quantification of hepatic iodide uptake ex vivo (Supplemental Fig. 2A) and qPCR analysis of hepatic NIS mRNA expression (Supplemental Fig. 2B), which revealed a 16-fold-lower hepatic NIS mRNA expression after injection of $\mathrm{dc}_{300} \mathrm{Ad5}$-CMV/NIS as compared with Ad5-CMV/NIS.

Analysis of liver enzymes after injection of Ad5-CMV/NIS $(n=5)$ revealed an alanine aminotransferase increase of $120 \%$ and a strong aspartate aminotransferase increase of approximately $400 \%$, which was significantly decreased after coating of the

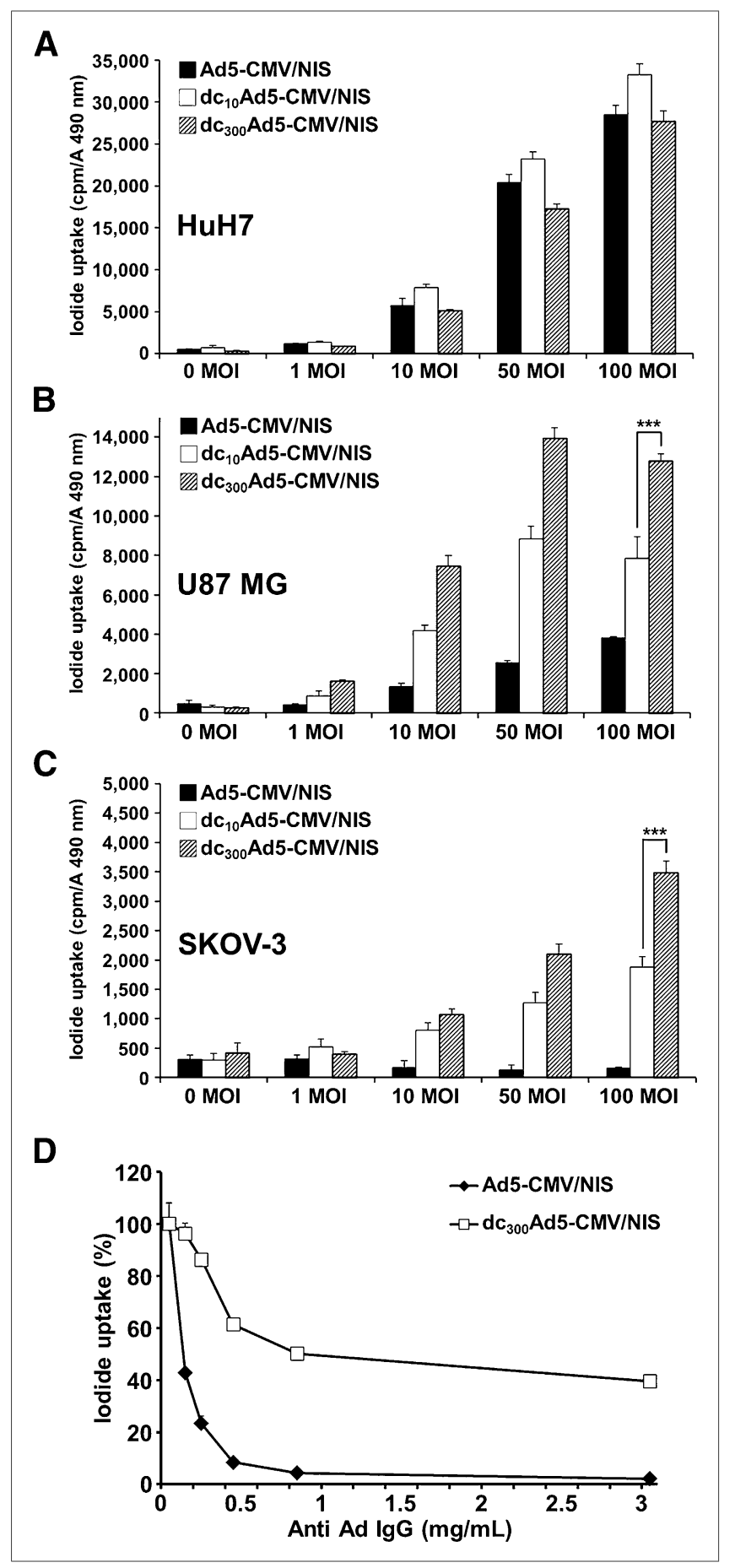

FIGURE 1. In vitro iodide uptake studies and neutralization assay with $\mathrm{dc}_{10} \mathrm{Ad5}$-CMV/NIS or $\mathrm{dc}_{300} \mathrm{Ad5}$-CMV/NIS demonstrated retained transduction efficacy in CAR-positive cells $(A)$ and significantly enhanced transduction efficacy in low-CAR-level cells (B) or CAR-negative cells $(C)$, thereby indicating CAR-independent uptake mechanisms of dendrimer-coated adenovirus. lodide uptake activity of cells infected with Ad5-CMV/NIS was progressively abolished by preincubation of virus with increasing amounts of anti-Ad5 antiserum, whereas infection with $\mathrm{dc}_{300} \mathrm{Ad5}-\mathrm{CMV} / \mathrm{NIS}$ showed reduced decrease in iodide uptake activity, suggesting effective antibody protection (D). ${ }^{* \star *} P \leq 0.001$. 


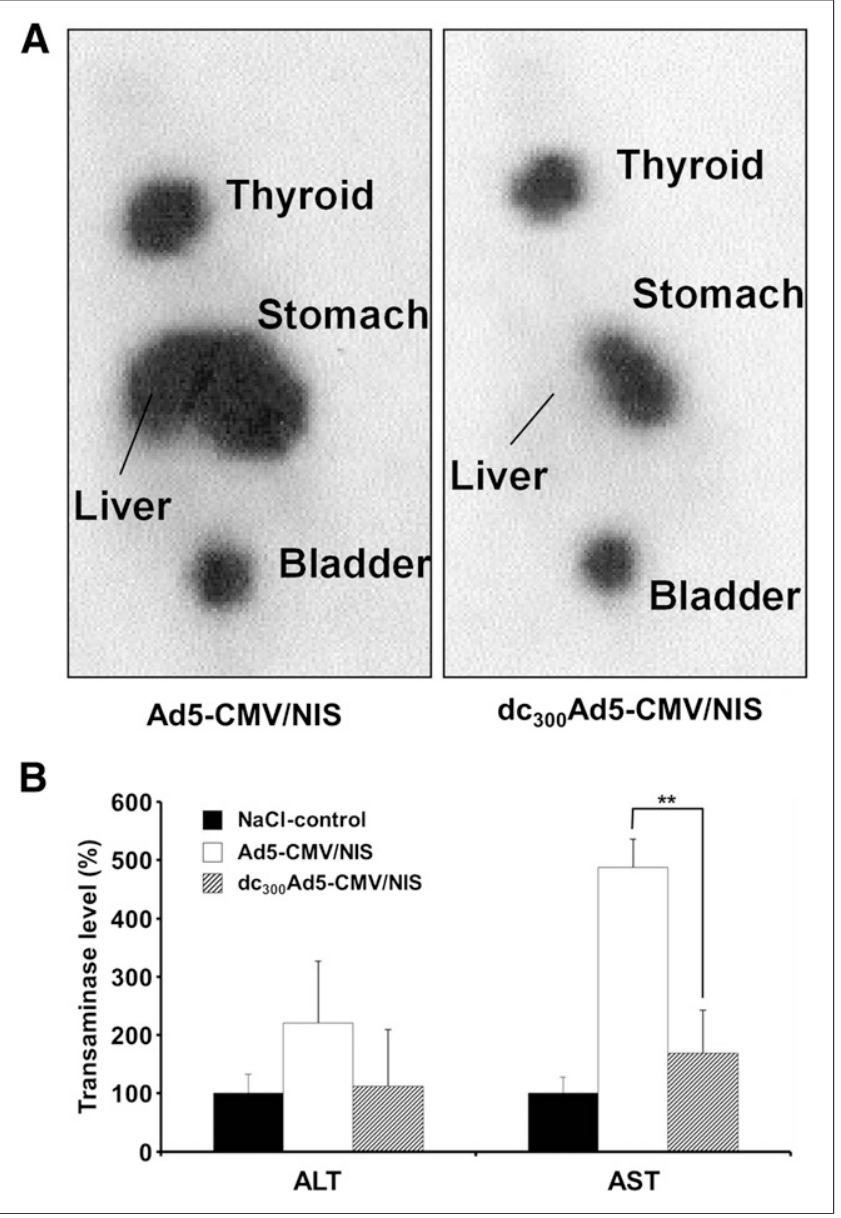

FIGURE 2. In vivo iodide uptake studies and analysis of liver toxicity. (A) Livers of mice accumulated high levels of radioiodine after intravenous injection of uncoated Ad5-CMV/NIS as shown by ${ }^{123}$ | $\gamma$-camera imaging (left), which was markedly reduced after systemic injection of coated adenovirus (right). (B) Injection of Ad5$\mathrm{CMV} / \mathrm{NIS}$ led to increased levels of serum alanine aminotransferase (ALT) and aspartate aminotransferase (AST) as compared with mice treated with saline only; these increased serum levels were mostly avoided by coating of the adenovirus before systemic administration. ${ }^{\star \star} P \leq 0.01$.

adenovirus before systemic administration $(n=5)$ (Fig. 2B). Liver tissue correspondingly showed significantly increased fatty degeneration after injection of Ad5-CMV/NIS, which was not observed after injection of $\mathrm{dc}_{300} \mathrm{Ad} 5-\mathrm{CMV} / \mathrm{NIS}$ (Supplemental Fig. 2C).

After intravenous injection of $\mathrm{dc}_{300} \mathrm{Ad} 5-\mathrm{CMV} / \mathrm{NIS}(n=9)$ into mice bearing subcutaneous $\mathrm{HuH} 7$ xenografts, tumoral radioiodine accumulation was approximately $13 \% \mathrm{ID} / \mathrm{g}{ }^{123} \mathrm{I}$ (biologic half-life, 3.5 h) (Figs. 3A, right, and 3B), whereas NIS-mediated radioiodine uptake in the liver was significantly reduced after dendrimer coating (Fig. 3A, right) as compared with injection with the uncoated vector (Fig. 3A, left). When a tumor mass of $1 \mathrm{~g}$ and an effective half-life of $3 \mathrm{~h}$ for ${ }^{131} \mathrm{I}$ were considered, a tumor absorbed dose of approximately $91 \mathrm{mGy} / \mathrm{MBq}$ of ${ }^{131} \mathrm{I}$ was calculated. In contrast, mice injected with the uncoated vector $(n=10)$ showed only low tumoral iodide accumulation above background level ( $\sim 3.5 \%$ ID/g, Fig. 3B). No additional uptake was observed in other nontarget organs such as the lung, spleen, or kidneys.

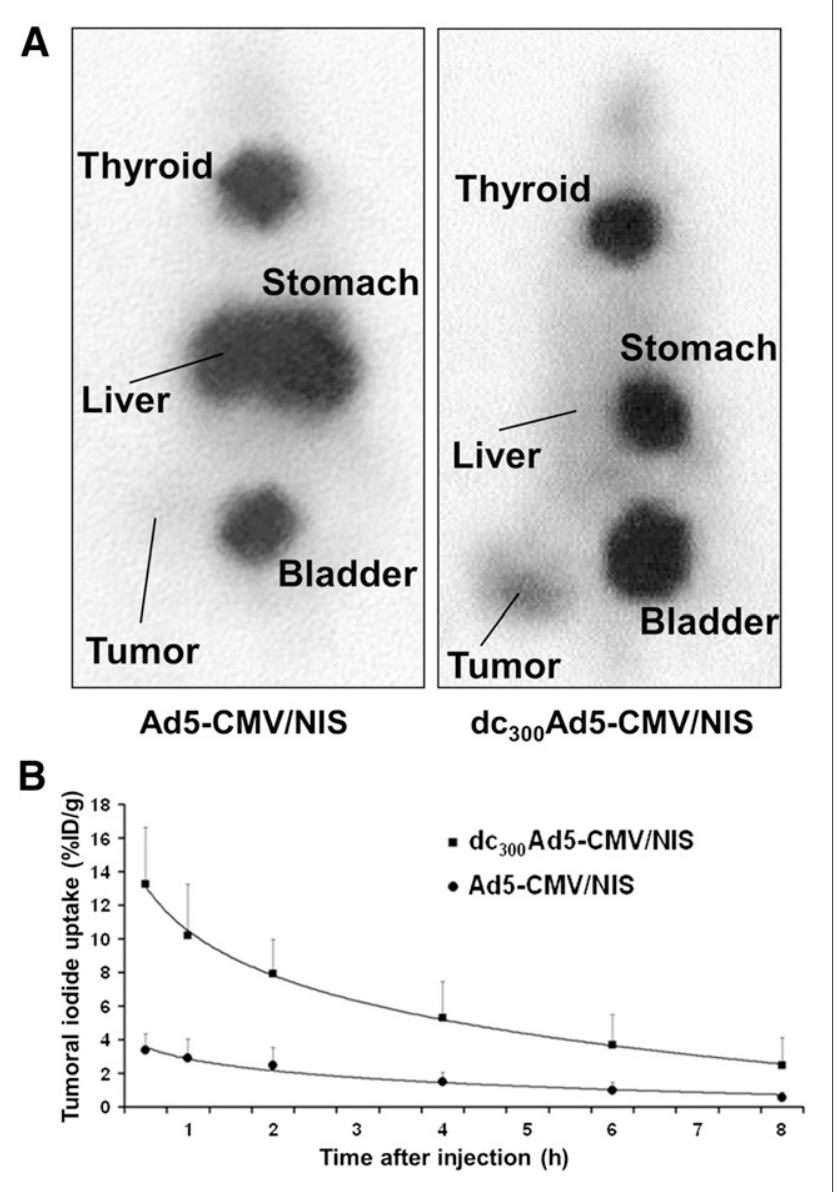

FIGURE 3. In vivo iodide uptake studies in tumor-bearing mice. (A) In vivo experiments showed high levels of radioiodine accumulation in liver of mice bearing $\mathrm{HuH} 7$ xenografts after intravenous injection of uncoated Ad5-CMV/NIS as shown by $\gamma$-camera imaging (left), which was significantly reduced after systemic injection of coated virus particles (right). Evasion from liver pooling of adenovirus resulted in increased tumoral transduction as seen by significantly higher iodide uptake activity of HCC xenografts (A, right, and B).

\section{Immunofluorescence and qPCR Analysis}

qPCR analysis of hepatic NIS mRNA expression revealed a 28fold decrease after intravenous injection of $\mathrm{dc}_{300} \mathrm{Ad} 5-\mathrm{CMV} / \mathrm{NIS}$ $(n=9)$ as compared with injection of Ad5-CMV/NIS $(n=10)$ (Fig. 4A, upper). Moreover, a significant 10-fold increase of NIS mRNA expression above background level was induced in $\mathrm{HuH} 7$ tumors after systemic injection of $\mathrm{dc}_{300} \mathrm{Ad} 5-\mathrm{CMV} / \mathrm{NIS}$ (Fig. 4B, upper). In contrast, tumoral NIS mRNA expression of mice injected systemically with Ad5-CMV/NIS was only 2.5 times higher than in untreated tumors. The NIS mRNA expression in the lung, spleen, and kidneys of mice showed no significant increase comparable to saline-treated mice (data not shown).

Immunofluorescence staining showed high levels of NISspecific immunoreactivity in the livers of mice after systemic injection of Ad5-CMV/NIS (Fig. 4A, lower, left), whereas livers of mice injected with $\mathrm{dc}_{300} \mathrm{Ad} 5-\mathrm{CMV} / \mathrm{NIS}$ showed only low levels of hepatic immunoreactivity (Fig. 4A, lower, right). Staining of $\mathrm{HuH} 7$ tumors revealed pronounced NIS-specific immunoreactivity after systemic injection of $\mathrm{dc}_{300} \mathrm{Ad} 5-\mathrm{CMV} / \mathrm{NIS}$ 


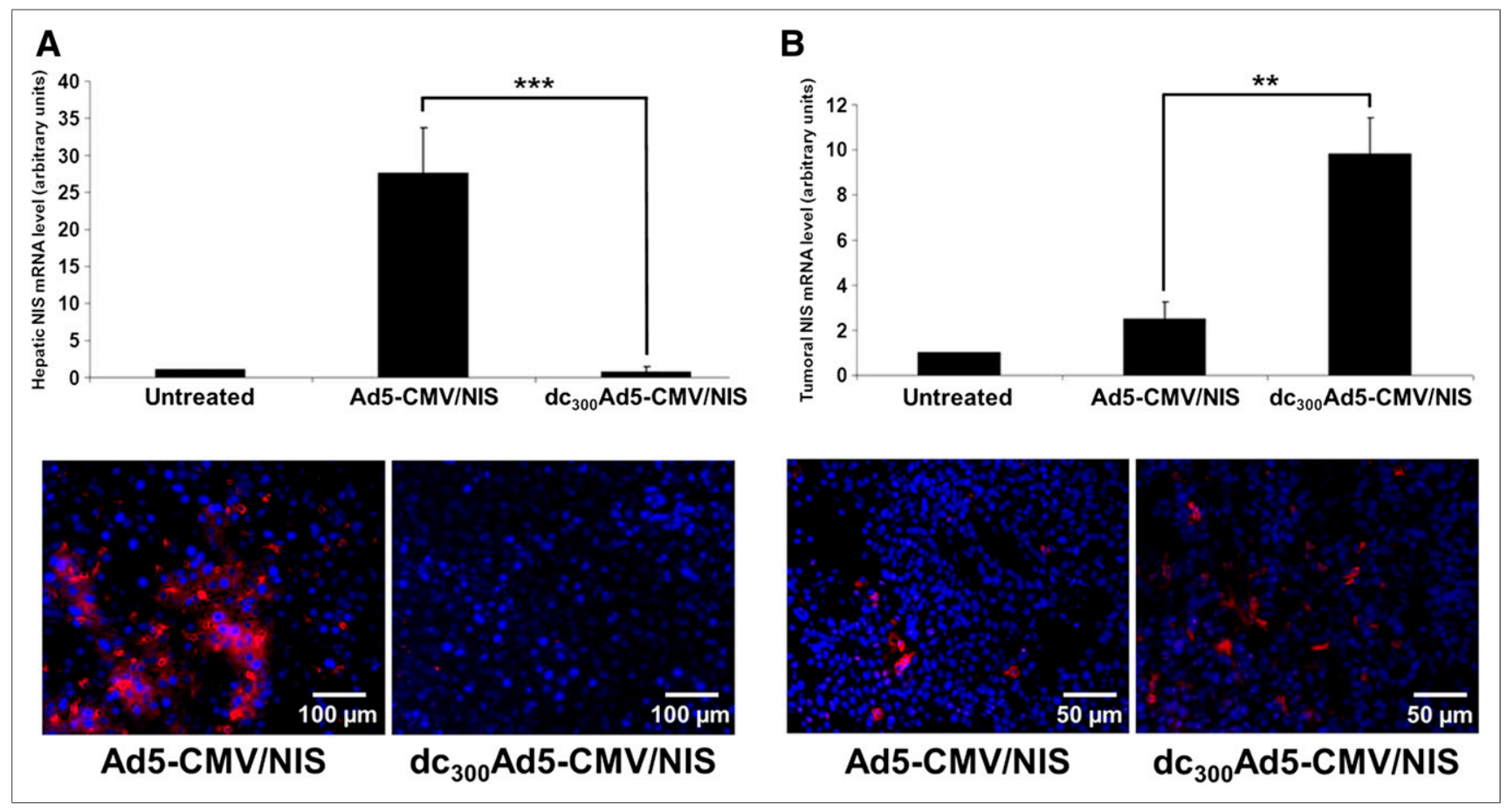

FIGURE 4. Immunofluorescence staining and qPCR analysis. (A) Analysis of hepatic NIS mRNA expression revealed 28-fold decrease after systemic injection of coated virus particles as compared with uncoated adenoviruses (upper). Immunofluorescence staining showed high levels of primarily membrane-associated NIS-specific immunoreactivity in livers of mice, systemically injected with Ad5-CMV/NIS (lower, left). In contrast, livers of mice injected with $\mathrm{dc}_{300} \mathrm{Ad5}-\mathrm{CMV} / \mathrm{NIS}$ showed only low levels of immunoreactivity (lower, right). (B) Significantly increased levels of NIS mRNA expression were induced in $\mathrm{HuH7}$ tumors after systemic NIS gene transfer when dc $300 \mathrm{Ad5}$ CMV/NIS was used as compared with injection of Ad5-CMV/NIS (upper). After systemic injection of dendrimer-coated adenoviruses, tumors revealed high NIS-specific immunoreactivity (lower, right) as compared with low levels after injection of uncoated adenoviruses (lower, left). ${ }^{* \star *} P \leq 0.001 .{ }^{* *} P \leq 0.01$.

(Fig. 4B, lower, right), which was significantly reduced after injection of Ad5-CMV/NIS (Fig. 4B, lower, left).

\section{Serial Imaging}

Pronounced tumoral iodide uptake was detected up to 15-18 d after a single intravenous injection of the conditionally replicating $\mathrm{dc}_{300} \mathrm{Ad} 5-\mathrm{E} 1 / \mathrm{AFP}-\mathrm{E} 3 / \mathrm{NIS}(n=7)$ (Fig. 5A). In contrast, only low tumoral iodide accumulation even after several days was observed in mice injected with Ad5-E1/AFP-E3/NIS $(n=6)$ (Fig. 5B). After injection of uncoated virus, serial scanning had to be stopped at day 11 because of excessive tumor growth (Fig. 5B). In contrast, tumors of mice injected with the coated adenovirus grew more slowly because of viral replication (Fig. 5A) and therefore allowed measurement beyond day 11. Regions of interest were quantified and confirmed significantly higher levels of tumor-specific iodide accumulation after application of coated adenovirus with a maximum at day 3 as compared with injection of uncoated virus (Supplemental Fig. 3). The most pronounced difference was seen at day 11, when $\mathrm{HuH} 7$ xenografts of mice injected with Ad5-E1/ AFP-E3/NIS accumulated only 1-2\%ID/100 $\mathrm{mm}^{3}$ at $2 \mathrm{~h}$ after injection of ${ }^{123} \mathrm{I}$. In contrast, mice injected with $\mathrm{dc}_{300} \mathrm{Ad} 5-\mathrm{E} 1 /$ AFP-E3/NIS still showed distinct tumoral iodide uptake activity of $5-6 \% \mathrm{ID} / 100 \mathrm{~mm}^{3}$.

\section{Radionuclide Therapy}

Mice injected with saline only $(\mathrm{NaCl}$ control, $n=8)$ showed an exponential tumor growth and had to be killed within 2-3 wk after onset of the experiments (Fig. 6). After a single intravenous injection of the conditionally replicating Ad5-E1/AFP-E3/NIS $(n=10)$, a significant delay in tumor growth was observed when dendrimer-coated adenovirus was used (dc ${ }_{300}$ Ad5-E1/AFP-E3/ NIS, $n=10)$ as compared with injection of uncoated adenovirus (Ad5-E1/AFP-E3/NIS, $n=10$ ), which showed no therapeutic effect, thereby indicating higher levels of viable virus reaching the tumor after dendrimer coating (Fig. 6A). One mouse treated with $\mathrm{dc}_{300} \mathrm{Ad} 5$-E1/AFP-E3/NIS showed complete tumor regression after $36 \mathrm{~d}$ until the end of the observation period (Fig. 6B).

To further evaluate the therapeutic efficacy of combined radiovirotherapy, in 2 additional therapy groups Ad5-E1/AFP-E3/NIS and $\mathrm{dc}_{300} \mathrm{Ad} 5$-E1/AFP-E3/NIS were administered intravenously, followed by injection of a therapeutic dose ${ }^{131} \mathrm{I}$. Mice treated with Ad5-E1/AFP-E3/NIS and ${ }^{131} \mathrm{I}$ (Ad5-E1/AFP-E3/NIS plus ${ }^{131} \mathrm{I}$, $n=10$ ) showed a significant delay in tumor growth and prolonged survival as compared with saline treatment or virus treatment alone (Fig. 6). Remarkably, radiovirotherapy of mice injected with $\mathrm{dc}_{300} \mathrm{Ad5}$-E1/AFP-E3/NIS (dc ${ }_{300} \mathrm{Ad5}$-E1/AFP-E3/NIS plus ${ }^{131} \mathrm{I}$, $n=10$ ) showed a strongly enhanced therapeutic effect, as seen by significantly delayed tumor growth and extensively prolonged survival (Fig. 6).

\section{DISCUSSION}

As one of the oldest targets of molecular imaging and targeted radionuclide therapy, characterization of NIS as a novel reporter and suicide gene offers the possibility of NIS gene transfer in nonthyroidal tumors, followed by diagnostic and therapeutic 


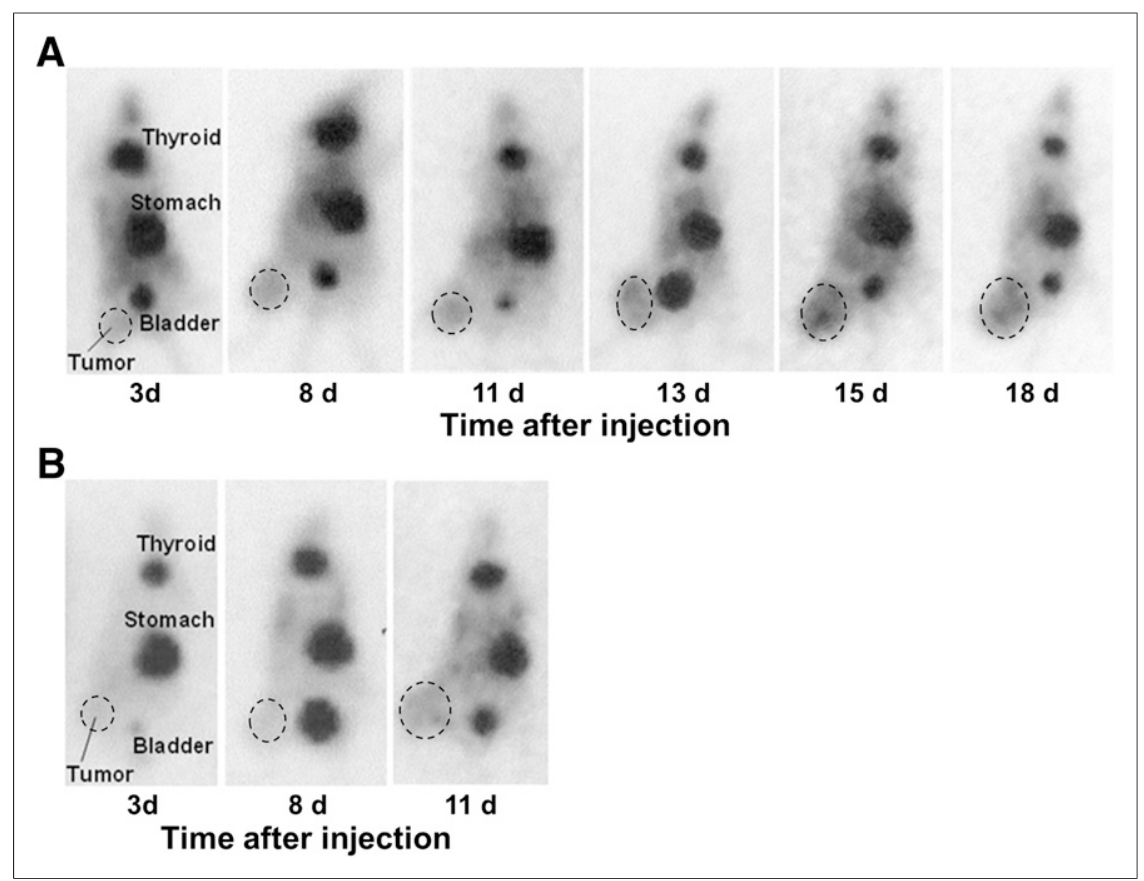

FIGURE 5. Serial ${ }^{123}$ I scintigraphy of mice bearing hepatoma xenografts after intravenous injection of Ad5-E1/AFP-E3/NIS with (A) or without (B) surface modification. Analysis of iodide uptake activity confirmed significantly increased levels of tumor-specific iodide accumulation after application of coated adenovirus $(A)$ as compared with injection of uncoated virus (B). Pronounced tumoral iodide uptake was detected up to 15-18 d after infection with dendrimer-coated adenovirus.

application of radioiodine (13). Our previous work convincingly demonstrated the proof of principle of the NIS gene therapy concept $(3,8,9,11,12,14,15)$. The next step toward clinical application has to be the development of gene-transfer vehicles that are able to promote targeted and efficient systemic NIS gene transfer with the potential to reach tumor metastases.

Species $\mathrm{C}$ adenoviruses have been shown to own high potential as vectors for gene transfer and for oncolytic virotherapy. However, after systemic application, wild-type adenovirus particles are rapidly cleared from the bloodstream into the liver tissue by specific molecular mechanisms, including virus-coagulation factor interaction and Kupffer cell trapping (1). Moreover, adenovirus infection relies on the widely expressed CAR on the cell surface, resulting in reduced tumor-selectivity (7). Further obstacles for successful systemic application of adenoviruses are the elimination by neutralizing antibodies and induction of immune and inflammatory responses (16). With the goal of systemic adenovirusmediated NIS gene delivery, in the current study we have explored the use of lower-generation PAMAM dendrimers (17) bearing terminal primary amino groups able to promote noncovalent electrostatic interaction with negatively charged amino acids on the external surface of the hexon protein of adenovirus serotype 5 in order to form a complex and provide efficient coverage.

To overcome the limited therapeutic potential of oncolytic virotherapy alone, adenoviruses may be armed with therapeutic genes, such as the NIS gene that allows for enhanced radionuclide therapy of tumor tissue in addition to oncolysis. Combination of adenovirus-mediated virotherapy and NIS-mediated radiotherapy is particularly attractive because adenoviruses are known to have a radiosensitizing effect (18), and at the same time ionizing radiation has been shown to generate an environment that is more susceptible to adenoviral transduction and replication $(19,20)$. Moreover, NIS gene therapy is associated with a substantial bystander effect based on the crossfire effect of the $\beta$-emitter ${ }^{131} \mathrm{I}$ with a path length of up to $2.4 \mathrm{~mm}$ that provides a powerful means to compensate for the limited tumor spread of viral vectors (21), thus enhancing the efficacy of virotherapy. A potential limitation of the NIS gene therapy concept is the endogenous NIS expression in the thyroid gland that, however, can effectively be downregulated by thyroid hormone pretreatment. The downregulation of this NIS expression is due to the exquisite regulation of thyroidal NIS by thyroid-stimulating hormone, as demonstrated in humans by Wapnir et al. (22). Another major argument that is frequently raised against the feasibility of using radioiodine therapy after NIS gene transfer into nonthyroidal tumors is the general assumption that organification of trapped radioiodine is a crucial prerequisite for effective radioiodine treatment due to increased retention of accumulated radioiodine. However, it has been shown that even thyroid carcinomas and their metastases often reveal a reduced capacity for iodide organification and thyroid hormone synthesis because of disrupted follicular architecture and function and lack of thyroglobulin expression $(23,24)$. Because accumulated radioiodine is not organified in $\mathrm{HuH} 7$ carcinoma cells, the data obtained using $\mathrm{HuH7}$ cell xenografts and presented in the article clearly indicate that iodide organification is not required to achieve a therapeutic effect of radioiodine in tumor tissue. In order to overcome the limitation of therapeutic efficacy by the lack of iodide organification, in the present study we aimed at combining NIS-mediated radioiodine therapy with virus-mediated oncolysis. Synergies between oncolytic virotherapy and NIS-mediated radioiodine treatment have been reported previously (2529). In a recent study, Haddad et al. (30) used an oncolytic vaccinia virus encoding the human NIS gene for local and systemic therapy of pancreatic carcinoma in a murine xenograft model. They demonstrated the feasibility of long-term serial imaging of tumoral NIS expression and enhanced therapeutic response after combination of oncolytic virotherapy and NIS-mediated radiotherapy. We previously illustrated the advantages of using oncolytic adenoviruses for successful realization of the NIS gene therapy approach (3). As a next step toward clinical application, here we are the first, to our knowledge, to show the feasibility of NIS-mediated radiovirotherapy using dendrimercoated replication-selective adenovirus vectors for systemic administration.

In the first steps of dendrimer-coated vector analysis, we have modified our replication-deficient adenovirus (Ad5-CMV/NIS) by PAMAM-G5 coating and have used NIS in its well-characterized function as reporter gene to monitor transduction efficiency and CAR selectivity (13).

We have been able to demonstrate the capacity of dendrimercoated Ad5-CMV/NIS to infect tumor cells with high efficiency 


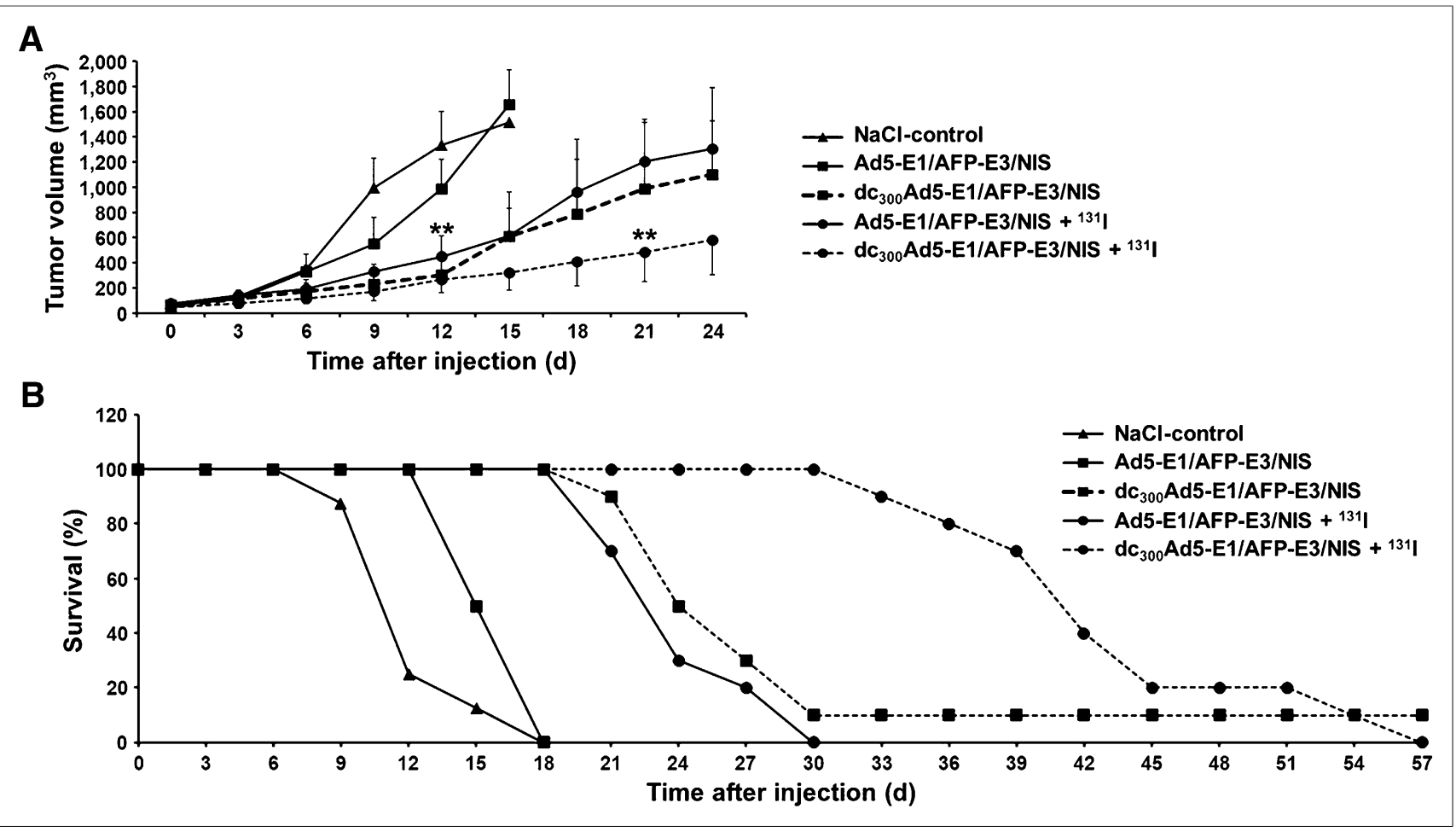

FIGURE 6. Therapeutic efficacy. (A, NaCl control) Radiovirotherapy studies in mice bearing HCC xenografts showed excessive tumor growth of control group treated with saline only. After a single intravenous injection of conditionally replicating Ad5-E1/AFP-E3/NIS (without surface modification), no therapeutic effect was observed. In contrast, intravenous injection of $\mathrm{dc}_{300}$ Ad5-E1/AFP-E3/NIS showed improved oncolytic efficacy. Intravenous application of Ad5-E1/AFP-E3/NIS followed by therapeutic dose of ${ }^{131}$ I revealed comparable delay in tumor growth. Intravenous injection of $\mathrm{dc}_{300} \mathrm{Ad5}$-E1/AFP-E3/NIS followed by additional application of ${ }^{131}{ }^{\prime}$ resulted in strongly enhanced therapeutic effect, as seen by significantly delayed tumor growth $(A)$ and prolonged survival $(B)$.

through CAR-independent uptake mechanisms, to form stable complexes in the presence of serum, and to protect the adenovirus, at least in part, from neutralizing antibodies (7). Our results are consistent with a study from Kasman et al., who reported polymerenhanced adenoviral transduction of CAR-negative cancer cells (31). By attachment of the positively charged polymer, the surface charge of the virus is inverted, thereby allowing it to bind to the cell surface (32). Subsequently, cellular transduction in our study was significantly enhanced, especially in the low-CAR or CARdeficient cell lines. Protection from neutralizing antibodies raises hope for a prolonged blood circulation time, as it was shown by Green et al. (33), with potential feasibility of repetitive applications in vivo. On the basis of our in vitro and in vivo data and the fact that both components of the complex were already applied in humans, clinical application of dendrimer-coated adenovirus is feasible and complexes will be stable in the bloodstream.

In vivo PAMAM-G5 coating of Ad5-CMV/NIS resulted in significantly lower hepatic accumulation of ${ }^{123} \mathrm{I}$ after systemic application. Evasion from liver pooling facilitated significantly enhanced radioiodine accumulation in hepatoma xenografts based on enhanced functional NIS expression. Hence, a tumor absorbed dose of $91 \mathrm{mGy} / \mathrm{MBq}$ for therapeutic ${ }^{131}$ I was calculated. In comparison, the study of Dingli et al. reported a comparable tumor absorbed dose of $108 \mathrm{mGy} / \mathrm{MBq}$ for ${ }^{131} \mathrm{I}$ after systemic injection of an NIS-encoding measle virus, which facilitated the elimination of tumors resistant to the virus alone (25). Serial ${ }^{123} \mathrm{I} \gamma$-camera imaging confirmed significantly increased levels of tumor-specific NIS-mediated iodide accumulation after application of the coated replication-selective $\mathrm{dc}_{300} \mathrm{Ad} 5$-E1/AFP-E3/NIS as compared with uncoated virus. Pronounced tumoral iodide uptake even after 15-18 d demonstrates high transduction efficiency and efficient replication of Ad5-E1/AFP-E3/NIS in the tumor. Evasion of the hybrid vector from scavenging by Kupffer cells could be the possible mechanism behind the observed liver detargeting of transgene expression, as it was suggested by the study of Prill et al. (34).

The enhanced permeability and retention effect is a phenomenon that describes the passive accumulation of macromolecules within the tumor stroma due to leaky tumor vasculature combined with inadequate lymphatic drainage (15) and is the basis for the observed passive tumor-targeting of dendrimer-coated adenovirus vectors after systemic delivery. Our dual-targeting strategy was further enhanced by an active transcriptional tumor-targeting using the alpha feroprotein promoter for tumor-selective replication and NIS expression. Effective liver detargeting furthermore resulted in a significantly lower extent of adenovirus-related liver toxicity in mice injected intravenously with dendrimer-coated adenovirus as shown by analysis of serum liver enzymes and liver histology, which is in agreement with previous studies $(33,35)$.

Therapy studies demonstrated that coating of the replicationselective Ad5-E1/AFP-E3/NIS before systemic administration significantly delayed tumor growth and extended survival as compared with injection of the uncoated adenovirus. Most importantly, the combined radiovirotherapy using the dendrimer-coated Ad5-E1/AFP-E3/NIS followed by a single application of a therapeutic dose of ${ }^{131} \mathrm{I}$ resulted in a strong further stimulation of the 
therapeutic effect, as seen by extensively delayed tumor growth and prolonged survival as compared with virotherapy alone or with control groups that used the uncoated vector. Our data suggest that dendrimer coating of adenoviral vectors increases the level of viable virus reaching peripheral tumor tissues.

\section{CONCLUSION}

Taken together, our results indicate that noncovalent coating of adenoviral vectors with synthetic dendrimers shows considerable promise for effective adenovirus liver detargeting and tumor retargeting, taking advantage of the merging of nonviral and viral vector technology. It therefore has the potential to improve current systemic gene delivery and tumor-targeting strategies and represents an innovative strategy to optimize the efficacy and safety of systemic NIS gene delivery that allows imaging and radiovirotherapy of nonthyroidal cancers, exploiting synergies between oncolytic virotherapy and NIS-mediated radionuclide therapy.

\section{DISCLOSURE}

The costs of publication of this article were defrayed in part by the payment of page charges. Therefore, and solely to indicate this fact, this article is hereby marked "advertisement" in accordance with 18 USC section 1734 . This study was supported by a grant from the Deutsche Forschungsgemeinschaft SFB 824, a grant from the Wilhelm-Sander-Stiftung (2008.037.1), a grant from the Center for Nanoscience (CeNS), and the excellence cluster Nanosystems Initiative Munich. No other potential conflict of interest relevant to this article was reported.

\section{ACKNOWLEDGMENTS}

We are grateful to Sissy M. Jhiang (Ohio State University, Columbus, $\mathrm{OH}$ ) for supplying the full-length human NIS complementary DNA and to Richard D. Anderson (Viraquest, North Liberty, IA) for the synthesis of the adenovirus vectors. We also thank Florian Kreppel (University of Ulm, Ulm, Germany) for helpful discussions and providing the SKOV-3 cells and Peter B. Luppa (TU Munich, Munich, Germany) for blood serum analysis.

\section{REFERENCES}

1. Duffy MR, Parker AL, Bradshaw AC, Baker AH. Manipulation of adenovirus interactions with host factors for gene therapy applications. Nanomedicine (Lond). 2012;7:271-288.

2. Baril P, Martin-Duque P, Vassaux G. Visualization of gene expression in the live subject using the $\mathrm{Na} / \mathrm{I}$ symporter as a reporter gene: applications in biotherapy. Br J Pharmacol. 2010;159:761-771.

3. Grünwald GK, Klutz K, Willhauck MJ, et al. Sodium iodide symporter (NIS)mediated radiovirotherapy of hepatocellular cancer using a conditionally replicating adenovirus. Gene Ther. 2013;20:625-633.

4. Kreppel F, Kochanek S. Modification of adenovirus gene transfer vectors with synthetic polymers: a scientific review and technical guide. Mol Ther. 2008;16: 16-29.

5. Yao XL, Nakagawa S, Gao JQ. Current targeting strategies for adenovirus vectors in cancer gene therapy. Curr Cancer Drug Targets. 2011;11:810-825.

6. Laga R, Carlisle R, Tangney M, Ulbrich K, Seymour LW. Polymer coatings for delivery of nucleic acid therapeutics. J Control Release. 2012;161:537-553.

7. Vetter A, Virdi KS, Espenlaub S, et al. Adenoviral vectors coated with PAMAM dendrimer conjugates allow CAR independent virus uptake and targeting to the EGF receptor. Mol Pharm. 2013;10:606-618.

8. Spitzweg C, Dietz AB, O'Connor MK, et al. In vivo sodium iodide symporter gene therapy of prostate cancer. Gene Ther. 2001;8:1524-1531.

9. Spitzweg C, Zhang S, Bergert ER, et al. Prostate-specific antigen (PSA) promoterdriven androgen-inducible expression of sodium iodide symporter in prostate cancer cell lines. Cancer Res. 1999;59:2136-2141.
10. Unterholzner S, Willhauck MJ, Cengic N, et al. Dexamethasone stimulation of retinoic acid-induced sodium iodide symporter expression and cytotoxicity of 131-I in breast cancer cells. J Clin Endocrinol Metab. 2006;91:69-78.

11. Willhauck MJ, Sharif Samani BR, Gildehaus FJ, et al. Application of ${ }^{188}$ rhenium as an alternative radionuclide for treatment of prostate cancer after tumor-specific sodium iodide symporter gene expression. J Clin Endocrinol Metab. 2007;92:4451-4458.

12. Klutz K, Schaffert D, Willhauck MJ, et al. Epidermal growth factor receptortargeted ${ }^{131}$ I-therapy of liver cancer following systemic delivery of the sodium iodide symporter gene. Mol Ther. 2011;19:676-685.

13. Spitzweg C, Morris JC. The sodium iodide symporter: its pathophysiological and therapeutic implications. Clin Endocrinol (Oxf). 2002;57:559-574.

14. Klutz K, Russ V, Willhauck MJ, et al. Targeted radioiodine therapy of neuroblastoma tumors following systemic nonviral delivery of the sodium iodide symporter gene. Clin Cancer Res. 2009;15:6079-6086.

15. Klutz K, Willhauck MJ, Dohmen C, et al. Image-guided tumor-selective radioiodine therapy of liver cancer after systemic nonviral delivery of the sodium iodide symporter gene. Hum Gene Ther. 2011;22:1563-1574.

16. Kang E, Yun CO. Current advances in adenovirus nanocomplexes: more specificity and less immunogenicity. BMB Rep. 2010;43:781-788.

17. Navarro G, Maiwald G, Haase R, et al. Low generation PAMAM dendrimer and $\mathrm{CpG}$ free plasmids allow targeted and extended transgene expression in tumors after systemic delivery. J Control Release. 2010;146:99-105.

18. Hart LS, Yannone SM, Naczki C, et al. The adenovirus E4orf6 protein inhibits DNA double strand break repair and radiosensitizes human tumor cells in an E1B-55K-independent manner. J Biol Chem. 2005;280:1474-1481.

19. Advani SJ, Mezhir JJ, Roizman B, Weichselbaum RR. ReVOLT: radiationenhanced viral oncolytic therapy. Int J Radiat Oncol Biol Phys. 2006;66: 637-646.

20. Hingorani M, White CL, Zaidi S, et al. Radiation-mediated up-regulation of gene expression from replication-defective adenoviral vectors: implications for sodium iodide symporter gene therapy. Clin Cancer Res. 2008;14:4915-4924.

21. Dingli D, Diaz RM, Bergert ER, O'Connor MK, Morris JC, Russell SJ. Genetically targeted radiotherapy for multiple myeloma. Blood. 2003;102:489-496.

22. Wapnir IL, Goris M, Yudd A, et al. The $\mathrm{Na}+/ \mathrm{I}-$ symporter mediates iodide uptake in breast cancer metastases and can be selectively down-regulated in the thyroid. Clin Cancer Res. 2004;10:4294-4302.

23. Mandell RB, Mandell LZ, Link CJ Jr. Radioisotope concentrator gene therapy using the sodium/iodide symporter gene. Cancer Res. 1999;59:661-668.

24. Valenta L. Metastatic thyroid carcinoma in man concentrating iodine without organification. J Clin Endocrinol Metab. 1966;26:1317-1324.

25. Dingli D, Peng KW, Harvey ME, et al. Image-guided radiovirotherapy for multiple myeloma using a recombinant measles virus expressing the thyroidal sodium iodide symporter. Blood. 2004;103:1641-1646.

26. Goel A, Carlson SK, Classic KL, et al. Radioiodide imaging and radiovirotherapy of multiple myeloma using VSV(Delta51)-NIS, an attenuated vesicular stomatitis virus encoding the sodium iodide symporter gene. Blood. 2007;110: 2342-2350.

27. Hakkarainen T, Rajecki M, Sarparanta M, et al. Targeted radiotherapy for prostate cancer with an oncolytic adenovirus coding for human sodium iodide symporter. Clin Cancer Res. 2009;15:5396-5403.

28. Peerlinck I, Merron A, Baril P, et al. Targeted radionuclide therapy using a Wnttargeted replicating adenovirus encoding the Na/I symporter. Clin Cancer Res. 2009;15:6595-6601.

29. Trujillo MA, Oneal MJ, McDonough S, Qin R, Morris JC. A probasin promoter, conditionally replicating adenovirus that expresses the sodium iodide symporter (NIS) for radiovirotherapy of prostate cancer. Gene Ther. 2010;17:1325-1332.

30. Haddad D, Zanzonico PB, Carlin S, et al. A vaccinia virus encoding the human sodium iodide symporter facilitates long-term image monitoring of virotherapy and targeted radiotherapy of pancreatic cancer. J Nucl Med. 2012;53:1933-1942.

31. Kasman LM, Barua S, Lu P, Rege K, Voelkel-Johnson C. Polymer-enhanced adenoviral transduction of CAR-negative bladder cancer cells. Mol Pharm. 2009;6:1612-1619.

32. Davis HE, Rosinski M, Morgan JR, Yarmush ML. Charged polymers modulate retrovirus transduction via membrane charge neutralization and virus aggregation. Biophys J. 2004;86:1234-1242.

33. Green NK, Herbert CW, Hale SJ, et al. Extended plasma circulation time and decreased toxicity of polymer-coated adenovirus. Gene Ther 2004;11:1256-1263.

34. Prill JM, Espenlaub S, Samen U, et al. Modifications of adenovirus hexon allow for either hepatocyte detargeting or targeting with potential evasion from Kupffer cells. Mol Ther. 2011;19:83-92.

35. Kim PH, Sohn JH, Choi JW, et al. Active targeting and safety profile of PEGmodified adenovirus conjugated with Herceptin. Biomaterials. 2011;32:23142326 . 